\section{GAMMA-RAY BURSTS}

\section{Huge explosion in the early Universe}

Long gamma-ray bursts (GRBs) are bright flashes of high-energy photons that can last for tens of minutes, they are generally associated with galaxies that have a high rate of star formation and probably arise from the collapsing cores of massive stars, which produce highly relativistic jets (collapsar model ${ }^{1}$ ). Here we describe $\gamma$ - and X-ray observations of the most distant GRB ever observed (GRB 050904): its redshift $\mathrm{f}^{2,3}(z)$ of 6.29 means that this explosion happened 12.8 billion years ago, corresponding to a time when the Universe was just 890 million years old, close to the reionization era'. This means that not only did stars form in this short period of time after the Big Bang, but also that enough time had elapsed for them to evolve and collapse into black holes.

GRB 050904 triggered the Burst Alert Telescope (BAT) on board the Swift ${ }^{5}$ satellite on 4 September 2005 at 1:51:44 GMT. The spacecraft quickly slewed to allow observations by

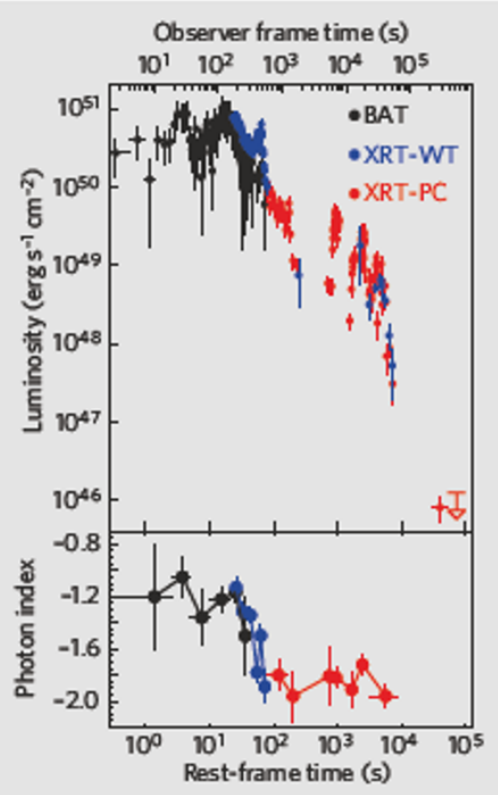

Figure 1 | Light curve and spectralevolution of GRB 050904 as observed by the Burst Alert Telescope (BAT) and the X-ray Telescope (XRT). WT, windowed timing mode data; PC, photon counting data. Top, evolution of the gamma-ray burst (GRB) $K$-corrected $0.2-10 \mathrm{keV}$ luminosity (the $K$-correction accounts for the redshift dependence of the luminosity in a given band of wavelengths). Error bars show $90 \%$ confidence. Times are referred to the BAT trigger. Rest-frame time is obtained by applying correction factor $(1+z)^{-1}$ to the observer frame time. Gaps in XRTPC data correspond to the part of the orbit when the satellite was not observing this GRB. Bottom, change in photon index $(\Gamma$, defined by the power law $F(E)=E^{r+1}$, where $F(E)$ is the observed flux of energy $E$ ) of GRB 050904 during the observation. Spectra were modelled using a power law with two absorbing components (galactic and intrinsic). the X-ray Telescope (XRT) ${ }^{67}$, which measured the burst for ten days after its onset. Figure 1 (top panel) shows the history of the burst. We shall present and discuss the GRB phenomenology from the point of view of the rest frame of its source.

The BAT light curve shows three main peaks: two of about $2 \mathrm{~s}$ at $T+3.8 \mathrm{~s}$ and $T+7.7 \mathrm{~s}$, and a long-lasting one at about $T+13.7 \mathrm{~s}$, where $T$ is the time of the burst onset. It also shows a weak peak at about $T+64 \mathrm{~s}$. The early XRT light curve shows a steep power-law decay with an index of $-2.07 \pm 0.03$; two flares are superimposed at $T+64 \mathrm{~s}$ (coincident with the last peak of the BAT light curve) and $T+170 \mathrm{~s}$. Although interrupted by the constraints of low-Earthorbit observation, the X-ray light curve reveals highly irregular intensity variations, probably due to the presence of flares for up to $T+1.5$ hours. At later times, flaring activity is not detected, leaving only a residual emission that is $10^{6}$ times lower than the initial intensity.

The flares in the XRT light curve can be interpreted as late internal shocks related to central engine activity. In this scenario, they would have the same origin as the first $\gamma$-ray emission $^{s-10}$, which would require the central engine to remain active for at least $5,000 \mathrm{sec}-$ onds, consistent with the collapsar model ${ }^{1}$.

Spectral analysis was performed by selecting time intervals corresponding to characteristic phases of the light curve evolution. All spectra were well modelled by a single power law, with both galactic and intrinsic absorption components in the case of the XRT spectra. Figure 1 (bottom) shows the evolution with time of the photon index $\Gamma$. The BAT spectra have $\Gamma=$ -1.2 . If we exclude the spectrum of the first XRT flare at $T+64 \mathrm{~s}$, the XRT photon indices show a clear, decreasing trend from about -1.2 to about -1.8 in the first $T+200 \mathrm{~s}$. No further spectral evolution is present in later XRT data.

The overall phenomenology of GRB 050904 is not peculiar with respect to other GRBs at lower redshift. This suggests that the mechanisms of GRB explosions in the early Universe and today are similar.

Based on the likely existence of population I/II stars in galaxies that were already metalenriched at these high redshifts" ${ }^{\text {, }}$, we expect about $10 \%$ of all bursts detected by Swift to be located at $z \geqslant 5$. A higher percentage would require an additional contribution to the high-redshift GRB population by metal-free population III stars, which are viable GRB progenitors for long-duration $\mathrm{GRBs}^{11}$. A more systematic search for GRB optical counterparts will increase the sample of these high-redshift GRBs, allowing us to probe the existence of metal-free massive stars of population III.

G. Cusumano', V. Mangano', G. Chincarin? ${ }^{2.3}$, A. Panaitescu', D. N. Burrows', V.La Parola',
T.Sakamoto ${ }^{67}$, S. Campana ${ }^{2}$, T. Mineo', G. Tagliaferri', L. Angelint', S. D. Barthelemy ${ }^{6}$, A.P. Beardmore, , P. T. Boyd ${ }^{6}$,L.R. Cominsky?, C. Gronwall', E. E. Fenimore ${ }^{4}$, N. Gehrels ${ }^{6}$, P. Giommi ${ }^{10}$, M. Goad ${ }^{3}$, K. Hurley ", J. A. Kennea ${ }^{5}$, K. O. Mason ${ }^{2}$, F. Marshall', P. Mészáros ${ }^{5,3}$, J. A. Nousek $k^{5}$, J.P.Osborne ', D.M. Palmer", P. W. A. Roming ${ }^{5}$, A. Wells ${ }^{8}$, N. E. White, B. Zhang ${ }^{14}$

'INAF-1stituto di Astrofisica Spazialee Fisica Cosmica di Palermo, 90146 Palermo, Italy e-mait: cusumano@ifcinatit

IINAF Osservator io Astronomico di Brera, 23807 Merate Italy;" "Università degili studi di Milano-Bicocca, Dipartimento difísica, $20126 \mathrm{M}$ ilan, Italy; ${ }^{4}$ Los A lamos National

Laboratory, PO Box 1663, Los Alamos, New Mexico 87545, USA: Depar tment of Astronomy \& Astrophysics and "Department of Physics, Pennsylvania State University, Philadelphia, Pennsylvania 16802, USA; ${ }^{6}$ NA SA/Goddard Space Flight Centec, Greenbelt, Maryland 20771, USA: ${ }^{7} \mathrm{National}$ Research Council, TI2114, Washington DC 20418, USA ; "Department of Physics and A stronomy, University of Leicester, Leicester LE1 7RH, UK; Department of Physics and Astronomy, Sonoma State Univer sity, Rohnert Park,

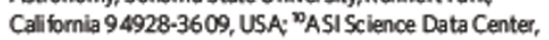
00044 frascati, Italy; "Space Sciences Laboratory, University of California, Berkeley, Cali fornia 94720-7450, USA: ${ }^{\square}$ Mullard Space Sclence Laboratory, University College London, Holmbury St Mary, Dorking, Surrey RH5 $6 \mathrm{NT}$, UK; "Depar tment of Physics, University of Nevada, Box 454002, Las Vegas, Nevada 89154-4002, USA

1. MacFadyen, A. L, Woosley, S. E \&Heger, A. Astrophys. L. $550,410-425$ (2001).

2. Tagliafer ri, G et al. Astron. Astraphys. 443, L1-L5 (2005)

3 Kawai, N etal. GCNCirc.3937 (2005).

4. Becker, R. H. et al. Astran L. 122, 2850-2857(2001)

5. Gehrels, N et al. Astraphys.J. 611, 1005-1020 (2004).

6. Sakamoto T.et al.GCN Circ 3938 (2005).

7. Mineo, T.et al. GCNCic. 3920 (2005).

8. Burrows, D.N.etal. Sdence 309, 1833-1835 (2005).

9. Zhang B.et d. Astraphys J. (in the press); preprint at $\checkmark h t t p: / /$ arXivorg/astro-ph/050832† (2005).

10. Nousek,J.A. et al. Astraphys. I (in the press); preprintat বhttp://arXivorg/astro-ph/0508332> (2005).

11. Bromm, V.\& Loeb, A. Astraphys J. (in thepress); preprint at বhttp://arXivorg/astro-ph/0509303> (2005).

Competing financial interests: declared none. Received 11 November 2005; accepted 23N November 2005. doi:10.1038/440164a

\section{CORRIGENDUM}

Developmental technology: Dogs cloned from adult somatic cells

Byeong Chun Lee, Min Kyu Kim, Goo Jang Hyun Ju Oh, Fibrianto Yuda, Hye Jin Kim,

M. Hossein Shamim, Jung Ju Kim, Sung Keun Kang, Gerald Schatten, WooSuk Hwang Nature 436, 641(2005)

Supplementary Table 1 of this communication has been replaced ( 9 March 2006; corrections shown in red) as the original peak values reported for two of the canine microsatellite markers (PEZO2, REN105L03) were in error, also, those for PEZ08 have been removed (further details are available from B.C.L. at bclee@snu.ac.kr). In the Table legend, the URL giving details of the markers has been updated. The patent applicationmentioned in the legend should originally have been declared as a competing financial interest. doi:10.1038/440164b

BRIEF COMMUNICATIONSARISING online www.nature.com/bca see Nature contents. 